\title{
The Responses of Visual Neurons in the Frontal Eye Field Are Biased for Saccades
}

\author{
Bonnie M. Lawrence ${ }^{1}$ and Lawrence H. Snyder ${ }^{2}$ \\ ${ }^{1}$ Department of Psychology, Case Western Reserve University, Cleveland, Ohio 44106, and 2Department of Anatomy and Neurobiology, Washington \\ University School of Medicine, St. Louis, Missouri 63110
}

Previous research suggests that visually responsive neurons in the frontal eye field (FEF) respond to visual targets even when they are not the goal of a saccadic eye movement. These results raise the possibility that these neurons respond to visual targets independent of the effector that is to be used to acquire the target locations. In the present study, we examined whether a plan to execute a saccade or a reach to a visual target influenced the response to and the representation of targets in the FEF. We recorded single unit responses to the onset of the target, during the delay period, and around the time of the movement, on interleaved saccade and reach trials of a delayed-response task. We found that the responses of approximately equal percentages of visual, visuomovement, and movement neurons (50\%, $58 \%$, and $58 \%$, respectively) were greater on saccade trials than on reach trials in at least one interval of the delayed-response task. Converse biases, in favor of reaches, were much less frequent (13\%, 10\%, and 19\%, in visual, visuomovement, and movement neurons respectively). Thus, although visual neurons may not be directly involved in triggering saccadic eye movements, they are nonetheless highly saccade-biased, with percentages comparable to neurons that are directly involved in triggering saccadic eye movements.

\section{Introduction}

The frontal eye field (FEF), located on the anterior bank of the arcuate sulcus in monkey, is involved in transforming sensory signals into motor commands for saccadic eye movements (for review, see Tehovnik et al., 2000). Within FEF there is a continuum of responses, from visual neurons that respond to the onset of a visual target in the receptive field, to movement neurons that respond around the time of a saccade (Bruce and Goldberg, 1985). Visual neurons remain tonically active when a delay is introduced between target presentation and saccade onset, suggesting that they are involved in the maintenance of spatial information (Funahashi et al., 1989; Lawrence et al., 2005). It is unclear, however, whether this maintenance occurs primarily or exclusively for saccades.

It is possible that FEF visual neurons are saccade biased, preferentially responding to and maintaining visual information for saccades relative to other purposes, such as reaches. Consistent with this possibility, Goldberg and Bushnell (1981) found enhanced responses from visual neurons when a target was the goal of a saccade compared to the object of a discrimination, and in at least one neuron, they found enhanced responses for a saccade compared to a reach. Because they did not use a delayed-response task, however, the extent to which this enhancement characterizes

Received May 14, 2009; revised Aug. 5, 2009; accepted Sept. 11, 2009.

The project described was supported by awards to L.H.S. (R01 EY012135) and to B.M.L. (F32 EY013898) from the National Eye Institute (NEI). The content is solely the responsibility of the authors and does not necessarily represent the official views of the NEl or the National Institutes of Health.

Correspondence should be addressed to Bonnie M. Lawrence, Department of Psychology, Mather Memoria Building, \#109, Case Western Reserve University, 10900 Euclid Avenue, Cleveland, OH 44106. E-mail: bonnie.lawrence@case.edu.

DOI:10.1523/JNEUROSCI.2352-09.2009

Copyright $\odot 2009$ Society for Neuroscience $\quad$ 0270-6474/09/2913815-08\$15.00/0 the maintenance of visual information is unknown. Mushiake et al. (1996) examined modulation in movement-related neurons and found that the presaccadic responses were uneffected by a simultaneous reach. While suggestive, these results do not rule out the possibility that reaches in the absence of a saccade drive FEF movement neurons. Moreover, because they examined only movement-related neurons, the extent to which these findings characterize visual neurons in FEF is not known.

It is also possible that visual neurons are not saccade biased. Consistent with this possibility, recent research confirms that visually responsive neurons in FEF respond to salient targets even when they are not the goal of a saccade (Thompson et al., 1997; Murthy et al., 2001; Sato et al., 2003). In a visual search task in which saccades are executed to an "oddball" target, visually responsive neurons signal the target before the saccade (Schall et al., 1995). However, this signal is not temporally correlated with the saccade onset (Thompson et al., 1996), occurs even when the saccade is mistakenly executed to a target other than the oddball stimulus (Murthy et al., 2001), and persists when the behavioral response is a lever press rather than a saccade (Thompson et al., 2005). These results suggest that visual neurons in FEF respond to and maintain visual targets in an unbiased manner.

We revisited this issue using a delayed-response paradigm in which the animal was required to look at or reach toward the remembered location only after a short memory period. We found biases for saccades that were comparable across cell types, including visual neurons. Thus, our data support a saccadespecific model of FEF.

\section{Materials and Methods}

Recording procedure. Animals were seated in a custom-designed monkey chair (Crist Instruments) with a fully open front that allowed for unconstrained arm movements to visual stimuli. Stimuli were back-projected 
by a CRT projector (Electrohome) onto a modified touch panel (Keytec) located $25 \mathrm{~cm}$ in front of the animal. Arm position was monitored using the touch panel, and eye position was monitored using a scleral search coil (CNC Engineering). The experiment took place in complete darkness in a soundattenuated room.

FEF recordings were made from two adult male Rhesus macaque (Macaca mulatta) monkeys. Recording chambers were placed flush with the skull $(25 \mathrm{~mm}$ anterior and $20 \mathrm{~mm}$ lateral, Horsley-Clarke coordinates), contralateral to the preferred hand. The determination of handedness, and as a result, chamber location, was based upon which hand the animal used more frequently early in training. Animals were viewed remotely to ensure the use of the dominant hand throughout a session. If, on the very rare occasion, the animal used the nondominant hand, lights were flashed to warn the animal, and the experiment resumed only when the animal reached for the fixation point with the dominant hand. Structural magnetic resonance imaging (MRI) was used to confirm the placement of each chamber with respect to the arcuate sulcus and also to localize recording sites [see the study by Lawrence et al. (2005) for recording site reconstruction].

Stimulation procedure. Neurons recorded within $200 \mu \mathrm{m}$ of sites at which electrical microstimulation of $<50 \mu \mathrm{A}$ evoked a consistent saccadic eye movement were defined as FEF neurons (Bruce et al., 1985). To make this determination, the animal began by fixating a blue target for $400 \mathrm{~ms}$. The fixation target was extinguished, and $100 \mathrm{~ms}$ later there occurred, with equal probability, a $70 \mathrm{~ms}$ interval of either stimulation or no stimulation. The fixation point reappeared $230 \mathrm{~ms}$ later on stimulation (biphasic, 250 microseconds/phase, $350 \mathrm{~Hz}$, $70 \mathrm{~ms}$ duration) and on no-stimulation trials. The animal was rewarded on every stimulation trial, and also on no-stimulation trials in which the eyes remained within $4.5^{\circ}$ of the extinguished fixation point. Because it is possible to elicit small eye movements at low thresholds in regions outside of FEF, only neurons recorded near stimulation sites resulting in perturbations greater than $2^{\circ}$ were used in the analyses. Significant perturbations ( $t$ test, $p<0.05$ ) ranged from $2.2^{\circ}$ to $28^{\circ}$, with a mean perturbation of $8.2 \pm 0.3^{\circ}$.

Receptive field mapping procedure. Spatially selective FEF neurons were then identified, and their receptive fields mapped, using a non-delayed center-out task. This task consisted of interleaved saccade trials, and combined saccade and reach trials. Movements were executed to eccentric stimuli presented in one of 8 possible directions, spaced $45^{\circ}$ apart, at a range of 3 eccentricities, for a total of 24 possible targets. This mapping determined the provisional "preferred" direction (the direction with the largest response) and the "null" direction (the direction with the smallest response, with the constraint that the null direction was $180^{\circ}$ from the preferred direction) based on the response of the neuron in the 100-200 $\mathrm{ms}$ interval following the onset of the target on the combined saccade and reach trials. From the responses obtained 100-200 ms after target appearance in the center-out movement task, and from the size of the stimulation-evoked eye movement, two target locations were chosen for further testing in the target-delay-cue paradigm (see below): one target in the preferred direction and one in the opposite (null) direction.

The preferred and null directions of spatially selective neurons were then confirmed using a target-delay-cue task. In this task, a target is presented, and following a delay period, a cue is presented signaling the effector (saccade or reach) in which the animal is to move to the target location (for additional details, see Lawrence and Snyder, 2006). The preferred direction of a neuron was defined as the direction associated with the largest response in any of the following three intervals of the target-delay-cue task: the early visual response (50-150 ms), the late

\section{Delayed Response Task}

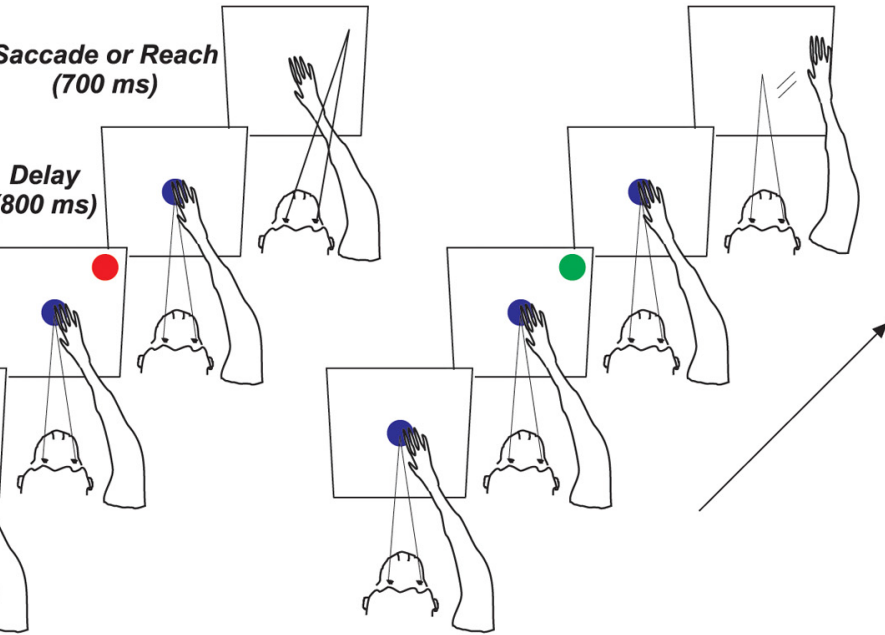

Reach Trials 

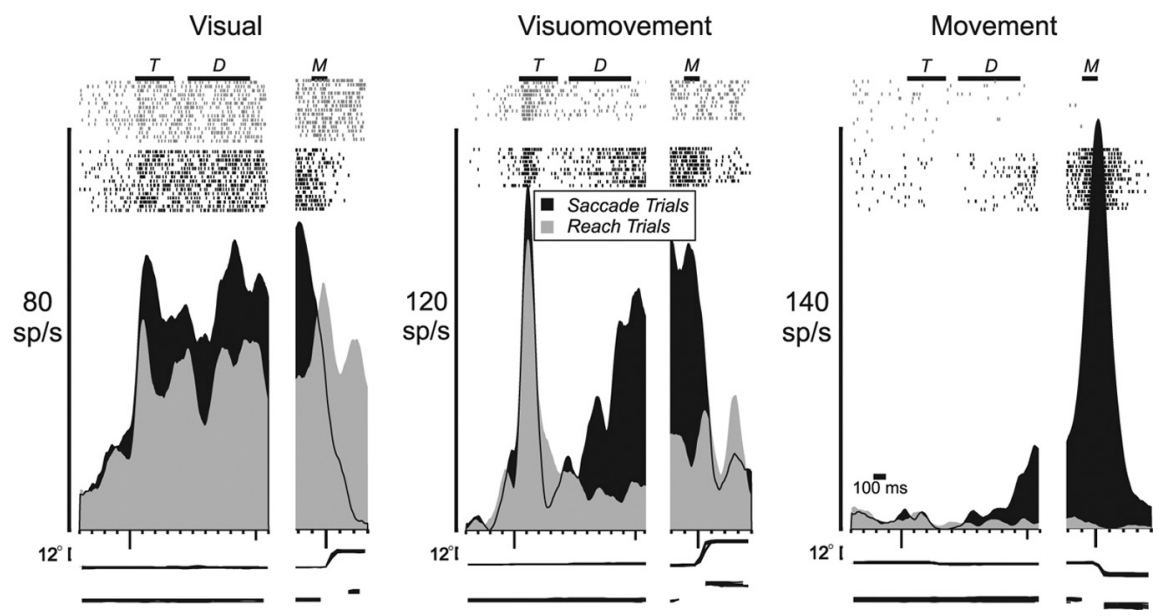

Figure 2. Activity of example visual (left panel), visuomovement (middle panel), and movement (right panel) neurons on saccade (black) and reach (gray) trials of the delayed-response task. Rasters and histograms representing data from each trial are aligned on the presentation of the target (left) or on the onset of movement (right). Each analysis epoch: target, delay, and movement (T, D, and $M$, respectively), is indicated. Horizontal eye position (top traces) and arm position (bottom traces) for each individual saccade and reach trial are represented at the bottom of the figure.

only because it provided the basis for the selection of RFs, but also because it contained a higher resolution mapping of preferred direction than the main task: in the center out task we collected data from 8 different target directions and 3 different target eccentricities for a total of 24 target locations. For each neuron, we calculated visual responses (interval 50 to $250 \mathrm{~ms}$ following the onset of the target) and movement responses (the $200 \mathrm{~ms}$ interval surrounding the onset of the movement) on saccade trials and on combined saccade and reach trials. Separate cosine tuning curves were then fit to the visual responses and the movement responses for each neuron for both saccade and combined saccade and reach trials (see supplemental Material, Fig. S2, available at www.jneurosci.org). We found that there was no significant difference in the tuning of the visual or movement responses (Watson's Two-Sample Test of Homogeneity $p>0.05$; median difference $=10.5^{\circ}$ and $19.6^{\circ}$, for visual and movement responses, respectively). Thus, the tuning on combined saccade and reach trials was not significantly different from the tuning on saccade trials.

Delayed-saccade and delayed-reach trials were divided into three intervals: target (50 to $350 \mathrm{~ms}$ interval following the onset of the target), delay (450 to $950 \mathrm{~ms}$ following the onset of the target), and movement (100 ms interval preceding the onset of the movement), unless otherwise noted. Paired $t$ tests were performed on raw spike counts for single unit and population data, comparing the activity on saccade and reach trials (in which the target appeared in the RF), for each of the intervals of the delayed-response task.

Detailed information on the classification of FEF neurons into visual, visuomovement, and movement cell types can be found in Lawrence et al. (2005). Briefly, for each neuron, the classification was based on the contrast ratio between visual and motor responses ([motor - visual]/ $[$ motor + visual $]$ ) on saccade trials of the delayed-response task for each neuron. Neurons with a contrast ratio of -1.0 had purely visual-related responses, while neurons with a contrast ratio of 1.0 had purely movement-related responses. Correlation was used to examine the relationship between the effector-bias in the response (saccade minus reach) and the visuomotor index. Separate correlations were performed for each interval of the delayed-response task (target, delay, movement).

Individual neurons with visuomotor indices ranging from -1.0 to -0.4 were classified as visual neurons, those with indices between -0.4 and 0.4 were classified as visuomovement neurons, and those with indices from 0.4 to 1.0 were classified as movement neurons. Separate one-way ANOVAs were then performed, one on each interval of the delayedresponse task, to determine whether effector biases differed across the three cell types. For ANOVAs with significant $F$ values, one-sample $t$ tests were performed to identify which cell types showed significant effector biases.

To determine whether the activity on saccade and reach trials into the $\mathrm{RF}$ diverged during the delayed-response task, the data were digitally low-pass filtered at $24 \mathrm{~Hz}$. Divergence was defined as the start of the first interval at which 25 consecutive pairs of points differed by at least 3 SEs from one another. For all other analyses, no filtering was used. For presentation purposes only, we low-pass filtered the neuronal data in Figures 2 ( $4 \mathrm{~Hz}$ low pass filter) and 4 (9 $\mathrm{Hz}$ low pass filter).

\section{Results \\ Single neurons}

The majority of single neurons in FEF demonstrated greater activity on saccade trials compared to reach trials during at least one interval of the delayed-response task. Examples of the activity of single visual, visuomovement, and movement neurons on saccade trials (black) and reach trials (gray) are presented in Figure 2. For each neuron, the activity is aligned both on the presentation of the target (left) and on the onset of the movement (right).

An example visual neuron, presented in Figure 2 (left panel), exhibited a burst of activity in response to the onset of the target with significantly greater activity on saccade trials than on reach trials $(53.33 \pm 6.15 \mathrm{sp} / \mathrm{s}$ and $35.33 \pm 5.24 \mathrm{sp} / \mathrm{s}$, respectively; mean $\pm \mathrm{SE}$; $t$ test, $p<0.05$; interval $100-200 \mathrm{~ms}$ following the onset of the target in the RF). This response was maintained throughout the delay period with significantly greater activity on saccade trials than on reach trials $(46.13 \pm 2.0 \mathrm{sp} / \mathrm{s}$ and $32.13 \pm 2.02 \mathrm{sp} / \mathrm{s}$, respectively; $p<0.0001$; interval $450-950 \mathrm{~ms}$ following the onset of the target in the RF). In the two-hundredmillisecond interval before the onset of the movement, the activity on saccade trials, but not on reach trials, decreased precipitously. In fact, there was no significant difference in activity on saccade and reach trials in the $100 \mathrm{~ms}$ interval before the onset of the movement into the RF $(41.33 \pm 5.33 \mathrm{sp} / \mathrm{s}$ and $50.00 \pm 4.14 \mathrm{sp} / \mathrm{s}$, respectively; $p>0.20$ ). Following the onset of movement into the $\mathrm{RF}$, the response reversed, with significantly greater activity on reach trials than on saccade trials $(32.00 \pm 3.80 \mathrm{sp} / \mathrm{s}$ and $1.0 \pm$ $0.72 \mathrm{sp} / \mathrm{s}$, respectively, $p<0.0001$, interval $200-400 \mathrm{~ms}$ following the onset of the movement into the RF). Thus, this visual neuron demonstrates an early bias for saccade targets that was maintained throughout the delay period but was reversed following the onset of the movement.

An example visuomovement neuron, presented in Figure 2 (middle panel), exhibited a similar burst of activity in response to target onset on saccade and reach trials $(85.0 \pm 9.57 \mathrm{sp} / \mathrm{s}$ and $71.0 \pm 10.80 \mathrm{sp} / \mathrm{s}$, respectively; $p>0.30$ ). Following the transient burst, there was an increase in activity during the delay period on saccade trials but not on reach trials. The delay period activity on saccade trials was significantly greater than the delay period activity on reach trials $(36.60 \pm 5.55 \mathrm{sp} / \mathrm{s}$ and $11.80 \pm 1.92 \mathrm{sp} / \mathrm{s} ; p<0.001)$. In the final $100 \mathrm{~ms}$ of the delay period, activity was $70.0 \pm 8.03$ $\mathrm{sp} / \mathrm{s}$ on saccade trials but only $13.0 \pm 3.35 \mathrm{sp} / \mathrm{s}$ on reach trials $(p<0.0001)$. Before the onset of the movement, activity remained significantly greater on saccade trials $(87.0 \pm 9.32 \mathrm{sp} / \mathrm{s})$ than on reach trials $(29.0 \pm 5.47 \mathrm{sp} / \mathrm{s} ; p<0.0001)$. Activity decreased precipitously with the onset of movement on saccade trials. Thus, this visuomovement neuron demonstrates a bias for saccades during the delay period and around the time of the movement.

An example movement neuron, presented in Figure 2 (right panel), was unresponsive on both saccade and reach trials after 
target onset $(5.33 \pm 2.15 \mathrm{sp} / \mathrm{s}$ and $6.00 \pm 2.35 \mathrm{sp} / \mathrm{s}$, saccade and reach trials respectively, $p>0.84)$ and during the early delay period $(3.67 \pm 1.24 \mathrm{sp} / \mathrm{s}$ and $1.33 \pm 0.77 \mathrm{sp} / \mathrm{s}$, saccade and reach trials respectively, $p>0.10$; interval $450-650 \mathrm{~ms}$ following the onset of the target in the RF). However, late in the delay period, a differential response emerged, with significantly greater activity on saccade trials than on reach trials $(10.33 \pm 2.51 \mathrm{sp} / \mathrm{s}$ and $2.00 \pm 0.82 \mathrm{sp} / \mathrm{s}, p<0.01$; interval $750-950 \mathrm{~ms}$ following the onset of the target in the RF). In the $100 \mathrm{~ms}$ interval before the onset of the movement into the RF, this neuron responded robustly on saccade trials but not at all on reach trials $(107.33 \pm$ $7.40 \mathrm{sp} / \mathrm{s}$ and $2.00 \pm 1.07 \mathrm{sp} / \mathrm{s}$, respectively; $p<0.0001$ ). Thus, this neuron demonstrates an overwhelming bias for saccades very late in the delay period and around the time of the movement.

Delayed-saccade and delayed-reach trials were divided into three intervals: target ( 50 to $350 \mathrm{~ms}$ interval following the onset of the target), delay (450 to $950 \mathrm{~ms}$ following the onset of the target), and movement (100 ms interval preceding the onset of the movement), unless otherwise noted. Table 1 presents the percentage (and number) of visual, visuomovement, and movement neurons that were significantly biased $(p \leq 0.05)$ for saccades $(\mathrm{S}>$ $\mathrm{R})$ or reaches $(\mathrm{R}>\mathrm{S})$ during any one interval of the delayedresponse task. We found that similar percentages of visual, visuomovement and movement neurons showed a significant bias for saccades over reaches $(50 \%, 58 \%$, and $58 \%$, respectively; left hand column) during at least one interval of the delayedresponse task. While a few cells showed the reverse pattern, that is, significantly greater responses on reach compared to saccade trials (right-hand column), the number of cells with a significant reach bias was not greater than would be expected by chance for any of the three cell types (proportion test, all $p$ values $>0.5$ ). Table 2 further presents separately, for each interval of the delayed-response task, the percentage (and number) of visual, visuomovement, and movement neurons that were significantly biased (see "Significant", $p \leq 0.05$ ) for saccades or reaches. The percentage (and number) of neurons for which the saccade or reach bias did not reach significance is also presented (see "Nonsignificant").

As is evident in Table 2, in visual neurons, the response to the target and during the delay period was significantly greater on saccade trials than on reach trials in almost one-third of these neurons. The percentage of neurons with a significant bias for saccades decreased to less than one- fourth of all visual neurons around the time of the movement. In visuomovement neurons, the response to the onset of the target was significantly greater on saccade trials than on reach trials in just over one-tenth of all neurons. The percentage of neurons with a significant bias increased to slightly more than one-fourth of all visuomovement neurons during the delay period, and to almost one-half of all visuomovement neurons around the time of the movement. Movement neurons, by definition, were relatively nonresponsive to the onset of the target. Not surprisingly, then, there no neurons with significantly greater activity on saccade trials than on reach trials around the time of target onset. During the delay period, however, a significant bias for saccades developed in one-sixth of all movement neurons, and increased sharply around the time of
Table 1. The percentage (and number) of neurons with a statistically significant ( $p \leq 0.05)$ saccade $(S>R)$ or reach $(R>S)$ bias for visual, visuomovement, and movement neurons during any one interval of the delayed-response task

\begin{tabular}{lll}
\hline & \multicolumn{2}{l}{ Significant bias } \\
\cline { 2 - 3 } Cell type & $\mathrm{S}>\mathrm{R}$ & $\mathrm{R}>\mathrm{S}$ \\
\hline Visual $(N=30)$ & $50 \%(15)$ & $13 \%(4)$ \\
Visuomovement $(N=31)$ & $58 \%(18)$ & $10 \%(3)$ \\
Movement $(N=26)$ & $58 \%(15)$ & $19 \%(5)$ \\
\hline
\end{tabular}

Table 2. The percentage (and number) of visual, visuomovement, and movement neurons with a significant or a nonsignificant saccade $(S>R)$ or reach $(R>S)$ bias, presented separately for each interval of the delayed-response task

\begin{tabular}{|c|c|c|c|c|}
\hline \multirow[b]{2}{*}{ Cell type/interval } & \multicolumn{2}{|l|}{$S>R$} & \multicolumn{2}{|l|}{$R>S$} \\
\hline & Significant & Nonsignificant & Significant & Nonsignificant \\
\hline \multicolumn{5}{|l|}{ Visual $(N=30)$} \\
\hline Target & $26.7 \%(8)$ & $46.6 \%(14)$ & $0.0 \%(0)$ & $26.7 \%(8)$ \\
\hline Delay & $30.0 \%(9)$ & $43.3 \%(13)$ & $0.0 \%(0)$ & $26.7 \%(8)$ \\
\hline Movement & $23.3 \%(7)$ & $46.7 \%(14)$ & $13.3 \%(4)$ & $16.7 \%(5)$ \\
\hline \multicolumn{5}{|c|}{ Visuomovement $(N=31)$} \\
\hline Target & $12.9 \%(4)$ & $48.4 \%(15)$ & $9.7 \%(3)$ & $29.0 \%(9)$ \\
\hline Delay & $25.8 \%(8)$ & $29.0 \%(9)$ & $6.5 \%(2)$ & $38.7 \%(12)$ \\
\hline Movement & $48.4 \%(15)$ & $38.7 \%(12)$ & $3.2 \%(1)$ & $9.7 \%(3)$ \\
\hline \multicolumn{5}{|c|}{ Movement $(N=26)$} \\
\hline Target & $0.0 \%(0)$ & $46.2 \%(12)$ & $7.6 \%(2)$ & $46.2 \%(12)$ \\
\hline Delay & $15.4 \%(4)$ & $30.8 \%(8)$ & $15.4 \%(4)$ & $38.4 \%(10)$ \\
\hline Movement & $57.7 \%(15)$ & $30.8 \%(8)$ & $0.0 \%(0)$ & $11.5 \%(3)$ \\
\hline
\end{tabular}

Target
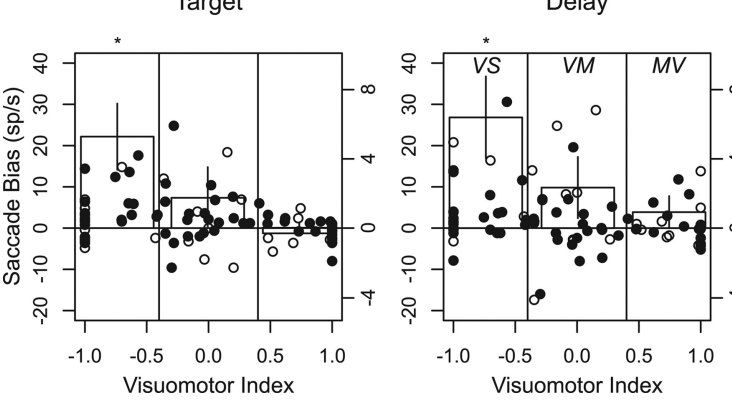

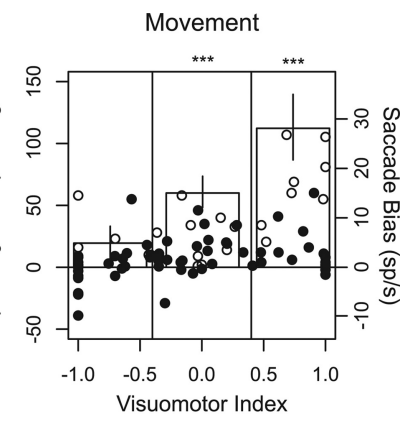

Figure 3. The response (saccade minus reach) of individual neurons plotted as a function of the visuomotor index, for the target (left panel), delay (middle panel), and movement (right panel) intervals of the delayed-response task. Filled and unfilled circles represent the responses of individual neurons from each monkey ( $M 1$ and $M 2$, respectively). For illustration purposes, one outlier 作 (visuomotor index $=-1.0$, effector bias value $=61 \mathrm{sp} / \mathrm{s}$ ). Vertical lines represent the boundaries for the visual (VS), visuomovement (VM), and movement (MV) cell types. Bars and error bars indicate the means and SEs, respectively, for each cell type; note the different scales for means (right-hand axis) compared to individuals (left-hand axis). ${ }^{*} p<0.05,{ }^{* * *} p<0.001$.

the movement to almost two thirds of all movement neurons. It should be noted that, because we were interested the activity that could be directly involved in triggering a movement, we quantified the movement-related response in the $100 \mathrm{~ms}$ interval before the onset of the movement (see Materials and Methods). Four movement neurons demonstrated a bias for saccades only after the onset of the saccade ( 300 to $500 \mathrm{~ms}$ following the onset of the movement) (see supplemental Material, Fig. S3, available at www.jneurosci.org as supplemental material, for an example neuron). As a result, these neurons were not included in the total percentage of movement neurons with saccade-biased responses during the movement interval.

To examine the relationship between cell type and saccade bias, we plotted the difference in activity between saccade and reach trials for individual neurons as a function of the visuomotor index, for target, delay, and movement intervals of the 
delayed-saccade task (Fig. 3). The visuomotor index was constructed by calculating the contrast ratio between visual and motor responses for each neuron (see Materials and Methods for additional details). Neurons with indices from -1.0 to -0.4 were classified as visual neurons (on the left of each panel), those with indices between -0.4 and 0.4 as visuomovement neurons (center of each panel), and those with indices from 0.4 to 1.0 as movement neurons (on the right of each panel). Data points above the horizontal line represent cells with a saccade bias, while points below the line represent cells with a reach bias. Filled and unfilled data points represent cells from M1 and M2, respectively.

Significant negative correlations were found between the saccade bias and the visuomotor index for the target and delay intervals ( $r=-0.25, p<0.05$, both intervals) as the saccade bias decreased from visual to movement neurons during these intervals, and a significant positive correlation was found for the movement interval as the saccade bias increased from visual to movement neurons $(r=0.36, p<0.001)$. For each of the three intervals, data were then binned by cell type (see superimposed bar graphs in Fig. 3) and subjected to an ANOVA. All three ANOVAs were significant (all $F_{(1,85)}>4.0$, all $\left.p<0.05\right)$. For the target and delay intervals, there was a significant saccade bias in visual neurons $\left(t_{(29)}>2.5, \mathrm{p}<0.05\right)$, but not in visuomovement $\left(t_{(30)}<1.35, p>0.30\right)$ or movement neurons $\left(t_{(25)}<1, \mathrm{p}>\right.$ $0.30)$. In contrast, for the movement interval, there was a significant saccade bias in visuomovement and movement neurons $\left(t_{(30)}=\right.$ $4.76, p<0.001, t_{(25)}=4.24, p<0.001$, respectively), but not in visual neurons $\left(t_{(29)}=1.43, p>0.15\right)$. Thus, the saccade bias to the onset of the target and during the delay period decreases from visual to movement neurons, while the saccade bias around the time of the movement increases from visual to movement neurons.

\section{Populations}

Population averaged responses on saccade trials (red) and reach trials (green) are presented in Figure 4 (see supplemental Material, Fig. S4, available at www.jneurosci.org as supplemental material, for the population averaged responses shown separately for each animal). The top panel shows that visual neurons are biased to respond to, and maintain, visual information for saccades. More specifically, there was significantly greater activity on saccade trials than on reach trials in response to the onset of the target (saccade minus reach: $5.28 \pm 1.91 \mathrm{sp} / \mathrm{s} ; t_{(29)}=2.76 ; p<$ 0.01 ) and during the delay period (saccade minus reach: $6.40 \pm$ $\left.2.37 \mathrm{sp} / \mathrm{s} ; t_{(29)}=2.70 ; p<0.05\right)$, but not around the time of the movement $\left(4.88 \pm 3.41 \mathrm{sp} / \mathrm{s} ; t_{(29)}=1.43 ; p>0.15\right)$. Following the movement into the RF (300 to $500 \mathrm{~ms}$ following the onset of the movement), the pattern of activity was reversed, with greater activity on reach trials than on saccade trials (saccade minus reach: $\left.-5.88 \pm 2.71 \mathrm{sp} / \mathrm{s} ; t_{(29)}=-2.17 ; p<0.05\right)$.

In contrast to the population of visual neurons, the population of visuomovement neurons (Fig. 4, middle panel) did not show a bias for saccades until late in the delay period. While the population of visuomovement neurons was responsive to target onset in the RF, there was no significant difference in the response on saccade and reach trials (saccade minus reach: $1.75 \pm 1.77$ $\left.\mathrm{sp} / \mathrm{s} ; t_{(30)}=0.99 ; p>0.30\right)$. In fact, visuomovement neurons $\mathrm{did}$ not become saccade biased until late in the delay period (saccade minus reach: $10.44 \pm 2.91 \mathrm{sp} / \mathrm{s} ; t_{(30)}=3.58 ; p<0.01 ; 950-1150$ $\mathrm{ms}$ interval following the onset of the target in the RF). These neurons were highly saccade biased around the time of the movement (saccade minus reach: $15.02 \pm 3.16 \mathrm{sp} / \mathrm{s} ; t_{(30)}=$ 4.76; $p<0.0001$ ). Following the movement into the RF, there
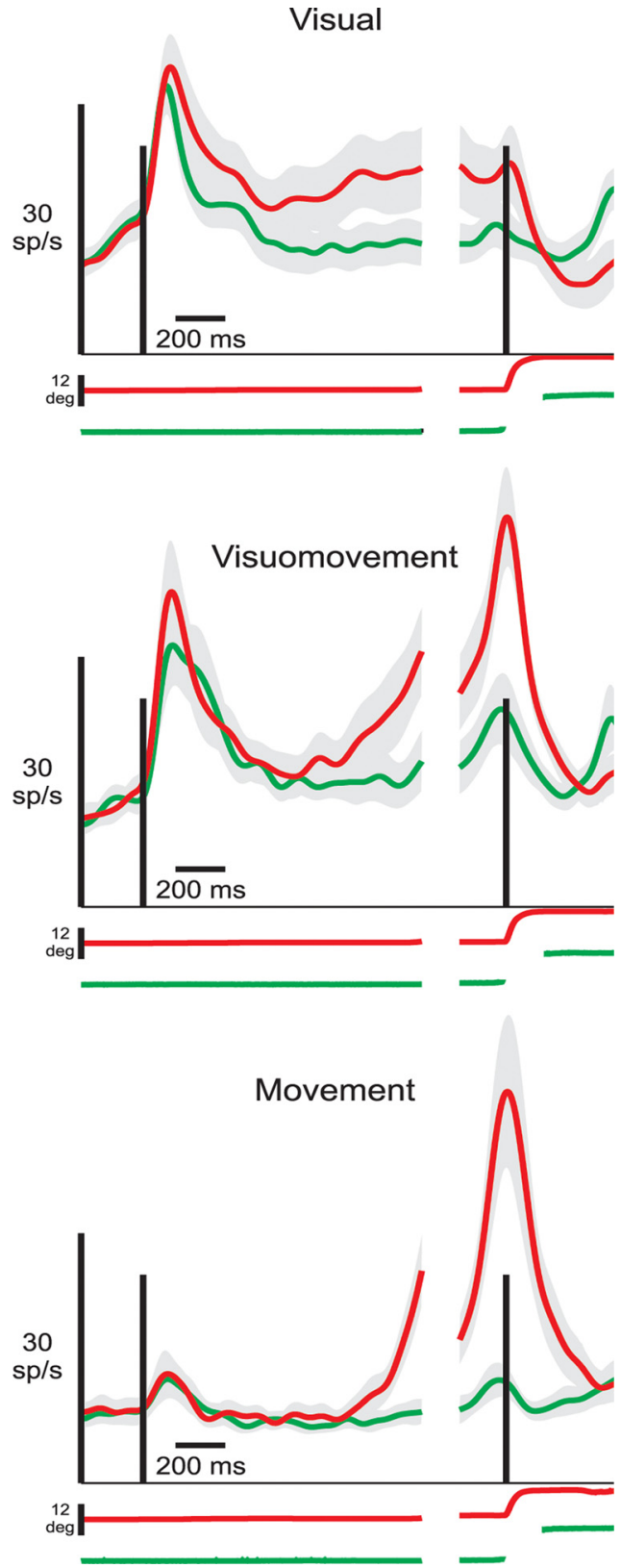

Figure 4. The population averaged responses on saccade (red) and reach (green) trials of the delayed-response task in visual (top), visuomovement (middle), and movement neurons (bottom). For each plot, data are aligned on the presentation of the target (left) or on the onset of movement (right). The gray shading indicates the SE for both types of trials. Horizontal eye position (top red traces) and arm position (bottom green traces) for saccade and reach trial, respectively, are represented at the bottom of each figure. Reach position is averaged across trials in which the animal maintained contact with the touch screen during the movement as well as those trials in which the animal did not maintain contact during the movement. For illustration purposes only, data were low-pass filtered (9 Hz low pass filter).

was no difference in the response on saccade and reach trials ( saccade minus reach: $-1.83 \pm 2.11 \mathrm{sp} / \mathrm{s} ; t_{(30)}=0.86 ; p<0.35$ ).

The population of movement neurons (Fig. 4, bottom panel) showed little task-related modulation until just before the onset of movement. Thus, there was no difference between saccade and reach trials in response to the presentation of the target (saccade minus reach; $\left.-0.32 \pm 0.61 \mathrm{sp} / \mathrm{s} ; t_{(25)}=-0.52 ; p>0.60\right)$, or during the delay period (saccade minus reach; $0.92 \pm 0.93 \mathrm{sp} / \mathrm{s} ; t_{(25)}=$ $0.99 ; p>0.33)$. A saccade bias emerged late in the delay period (interval 950 - $1150 \mathrm{~ms}$ following the onset of the target; saccade 
minus reach; $10.02 \pm 2.40 \mathrm{sp} / \mathrm{s} ; t_{(25)}=$ $4.17 ; p<0.001$ ), and increased dramatically around the time of the movement (saccade-reach; $28.11 \pm 6.62 \mathrm{sp} / \mathrm{s} ; t_{(25)}=$ 4.24 ; $p<0.0001$ ). Following the movement into the RF, the response on saccade and reach trials returned to baseline levels (saccade minus reach: $1.21 \pm 2.13 \mathrm{sp} / \mathrm{s}$; $t_{(25)}=0.57 ; p>0.50$ ).

A direct comparison of the time course of activity on saccade and reach trials confirmed these findings. On average, activity on saccade and reach trials first became biased for saccades in visual neurons 116 ms after the onset of the visual stimulus (see Materials and Methods for details of the analysis). Activity in visuomovement neurons, in contrast, did not show a sustained bias until $953 \mathrm{~ms}$ after target onset, or $147 \mathrm{~ms}$ before the end of the memory period. Similarly, a sustained bias did not emerge in movement neurons until 1016 $\mathrm{ms}$ after target onset. Thus, the responses of visual neurons became biased for saccades shortly after the onset of the visual target (Fig. 4, top panel). In contrast, the responses of visuomovement and movement neurons did not become biased for saccades until late in the delay period (Fig. 4, middle and bottom panels, respectively).

While the response around the time of the movement was biased for saccades, there was nonetheless an increase in activity just before reaches, especially in visuomovement neurons. This reach-related activity (in the $100 \mathrm{~ms}$ interval preceding the onset of the movement) was significantly greater on trials in which the reach was executed to a remembered target in the RF than out of the RF in visuomovement neurons (difference of $6.04 \pm 1.95$ $\mathrm{sp} / \mathrm{s} ; p<0.01)$, but not in visual $(1.77 \pm 1.59 \mathrm{sp} / \mathrm{s} ; p>0.25)$ or movement neurons $(1.52 \pm 2.03 \mathrm{sp} / \mathrm{s} ; p>0.45)$. While the functional significance of response around the time of the reach is difficult to interpret, these results suggest that the response in visuomovement neurons was spatially specific and was not due to nonspatial factors such as arousal.

\section{Discussion}

The present results demonstrate that visual neurons in FEF are biased to respond to and maintain visual targets for saccadic eye movements. Fifty percent of visual neurons were significantly more active on saccade trials than on reach trials during at least one interval of the delayed-response task, and only $13 \%$ of visual neurons showed the reverse effect (Table 1). These percentages are similar to the corresponding values for visuomovement and movement cells (58\% and $10 \%$, and $58 \%$ and $19 \%$, respectively). A strong bias for saccade compared to reach trials is also seen in the population-averaged responses of all three cell types, including visual neurons (Fig. 4). While the saccade bias in the population of visuomovement and movement neurons occurred primarily during the movement period, the saccade bias in the population of visual neurons emerged shortly after target onset and was maintained throughout the delay period. These results suggest that while visual neurons are not directly involved in triggering saccadic eye movements, they are nonetheless highly near-baseline levels (dashed lines).

\section{Visual Neurons}

Array

-Target

- Distractor

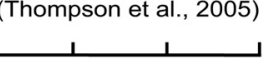

Target

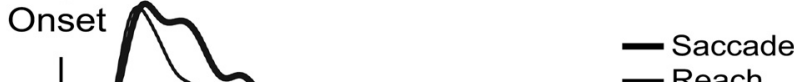

- Reach
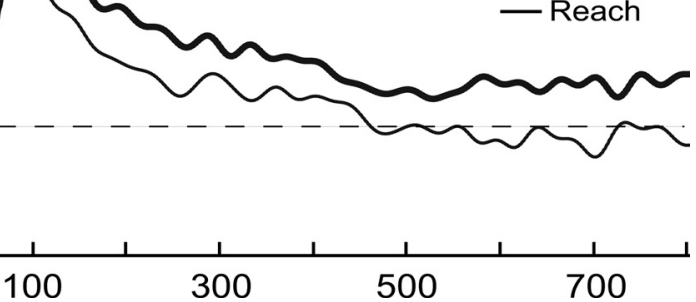

Figure 5. A comparison of the present results with those of Thompson et al. (2005), reprinted with permission. In both the study the duration of the task. In contrast, on distractor and reach trials (thin traces), the activity declined rapidly to baseline or

biased to respond to, and maintain, target locations for saccades compared to reaches.

\section{Relation to previous research}

The present results are consistent with the results of the classic study of Bruce and Goldberg (1985) which compared FEF responses during saccade and reach trials and concluded that "frontal eye field visual activity is best understood as providing targets for visually guided saccades, and not as participating in a more general analysis of the visual world." However, several recent lines of inquiry have suggested that visual neurons in FEF do play a more general role in visual processing. In particular, a number of studies have argued that visual neurons are involved in allocating visual attention to salient target locations independent of whether such locations are the goal of a saccade (Thompson et al., 1997, 2005; Murthy et al., 2001; Sato et al., 2003).

One such study, the results of which are reprinted in Figure 5 (top), found that visual neurons in FEF discriminate between a target (thick trace) and a distractor (thin trace), even when the required response to the target is a lever turn and not a saccade (Thompson et al., 2005). These results suggest that visual neurons do not preferentially respond to targets for upcoming saccades, but instead respond to any task-relevant target regardless of how that target will be used. In contrast, the results of the present study suggest that visual neurons preferentially respond to targets for upcoming saccades (Fig. 5, bottom). Indeed, the target-evoked activity on reach trials declined rapidly to baseline or near-baseline levels, despite the fact that the target was clearly important to the animal's performance of the task. Thus, the Thompson et al. (2005) study suggests that the response of a visual neuron in FEF is determined by whether or not the stimulus is task-relevant (i.e., salient), whereas the current study suggests that the response is determined by whether or not the 
stimulus is the target of an upcoming saccade. How can this discrepancy be resolved?

One possibility is that, despite being task relevant, attention is not allocated to reach targets (Bisley and Goldberg, 2003). The results of Deubel and Schneider (2003), however, suggest that attention is allocated to reach targets, at least initially (see also Deubel et al., 1998). They probed the locus of attention shortly after the cue to move in delayed-saccade and -reach trials. The discrimination of a probe away from the movement endpoint was impaired on saccade trials, consistent with the well known finding that attention is coupled to the saccade endpoint around the time of the movement (Shepherd et al., 1986; Hoffman and Subramaniam, 1995; Deubel and Schneider, 1996). This was not the case for long delayed reach trials $(>300 \mathrm{~ms})$. For these trials, a probe appearing $80 \mathrm{~ms}$ after a cue to initiate a reach was discriminated equally well at all locations, both at and away from the reach endpoint. These results suggest that attention is not sustained at reach targets. They are not conclusive, however, since they quantify attention as the movement is initiated and not during the delay period. Thus, while it is possible that monkeys do not attend to reach targets during a delay period and that visual neurons in FEF reflect the attended locations in space in both the current study and in Thompson et al. (2005), the evidence is inconclusive.

A related possibility is that attention is allocated to reach targets, but that the neuronal correlates of this attentional allocation are not found within the saccade system (FEF), but are found instead within the reach system (e.g., dorsal premotor cortex). Such a possibility would necessitate different attentional systems for different effectors. While this may seem improbable, Rizzolatti et al. (1994), in their formulation of the "premotor theory of attention," which hypothesizes that the circuitry for shifts of attention and goal-directed movements is one and the same, raise this very possibility. (A strict interpretation of the premotor theory of attention would dictate that the neuronal correlates of attentional allocation would be found in neurons that are thought to be directly involved in triggering a movement, for example, FEF movement neurons. The present results suggest instead that while the neuronal correlates of attentional allocation may be found within the circuitry of a movement system, they are not found in those neurons directly involved in triggering the movement.)

The premotor theory of Rizzolatti et al. (1994) hypothesizes that shifts of spatial attention before saccades result from the activation of the saccade circuitry, and similarly, that shifts of spatial attention before reaches result from the activation of the reach circuitry. This would necessitate, however, that the activity in visual neurons in Thompson et al. (2005) is saccade related. How could this be the case when a saccade was never executed to a target in their study? It is possible that the target in the visual search task used in Thompson et al. (2005) not only attracts attention but also evokes a plan for a saccade. In both the search and the reach task, the subject is explicitly and carefully trained to suppress the natural impulse to look at the target. A residual saccade plan may nonetheless remain. This plan may be stronger in the search task, where receiving a reward requires making a difficult discrimination. In contrast, target identification and localization in the reach task is quite easy, and therefore a plan to saccade to the target may be present only transiently. Thus, the results of both Thompson et al. (2005) and the current study could be explained if FEF visual neurons specifically encode the location of targets for saccades.

\section{Relation to other areas}

Previously, Snyder and colleagues have found that the responses of neurons in the lateral intraparietal area (LIP) and in the parietal reach region (PRR) of the parietal cortex are biased for planning saccades and reaches, respectively (Snyder et al., 1997; Quian Quiroga et al., 2006), and that there is a nonspatial component to these planning signals, since the bias appears even when the spatial target of the movement is not yet known (Calton et al., 2002; Dickinson et al., 2003). They hypothesized that LIP and PRR are nodes in a saccade and reach network, respectively, that also includes frontal and subcortical saccade centers (e.g., frontal eye fields, superior colliculus, cerebellum) and reach centers (e.g., dorsal premotor cortex) (Snyder et al., 2000). They further hypothesized that the relationship between areas within each network may be either hierarchical or distributed. Frontal cortex may elaborate nascent saccade and reach plans from the parietal cortex (a hierarchical or serial architecture), or frontal and parietal cortex may work in parallel to produce saccade and reach plans (a distributed architecture) (Snyder et al., 2000). Recently, we have shown that nonspatial saccade planning signals are present in FEF, but that neither the onset nor the magnitude of these signals are earlier, or larger, respectively, in frontal cortex than in parietal cortex, consistent with a distributed model (Lawrence and Snyder, 2006). The fact that saccades and reaches are coordinated suggests that saccade and reach networks interact. This interaction could begin as early as parietal cortex (Cohen and Andersen, 2002). Neurons in the frontal cortex, the supplementary eye field, the pre-supplementary motor area (pre-SMA), and the SMA all respond differently to isolated eye or arm movements compared to coordinated movements, but this is not true of FEF (Mushiake et al., 1996; Fujii et al., 2002). Although the present study was not designed to examine the interaction between saccades and reaches, the lack of FEF modulation during reach trials shown in the current study, combined with the results of Mushiake et al. (1996), suggests that saccade-reach interactions do not occur in the FEF.

The saccade bias in visual neurons in FEF could provide a "top-down" attention signal to earlier visual areas. Indeed, presaccadic enhancement of visual responses have been found in V4 (Fischer and Boch, 1981a,b; Tolias et al., 2001; Mazer and Gallant, 2003; Moore and Chang, 2009). Also consistent with top-down attentional modulation, Moore and Armstrong (2003) have found that stimulation of FEF neurons enhances visual responses in retinotopically corresponding locations of V4. It seems likely that V4 neurons would be similarly biased during a delay period before a saccade. Indeed, the results of recent functional MRI studies are consistent with a saccade bias in the extrastriate cortex of humans (Levy et al., 2007; Beurze et al., 2009). An interesting question for future research is whether V4 neurons are also enhanced during the delay period before a reach in the absence of a saccade. Such an effect would presumably be mediated by projections from areas other than FEF.

\section{References}

Beurze SM, de Lange FP, Toni I, Medendorp WP (2009) Spatial and effector processing in the human parietofrontal network for reaches and saccades. J Neurophysiol 101:3053-3062.

Bisley JW, Goldberg ME (2003) Neuronal activity in the lateral intraparietal area and spatial attention. Science 299:81-86.

Bruce CJ, Goldberg ME (1985) Primate frontal eye fields. I. Single neurons discharging before saccades. J Neurophysiol 53:603-635.

Bruce CJ, Goldberg ME, Bushnell MC, Stanton GB (1985) Primate frontal eye fields. II. Physiological and anatomical correlates of electrically evoked eye movements. J Neurophysiol 54:714-734. 
Calton JL, Dickinson AR, Snyder LH (2002) Non-spatial, motor-specific activation in posterior parietal cortex. Nat Neurosci 5:580-588.

Cohen YE, Andersen RA (2002) A common reference frame for movement plans in the posterior parietal cortex. Nat Rev Neurosci 3:553-562.

Deubel H, Schneider WX (1996) Saccade target selection and object recognition: evidence for a common attentional mechanism. Vision Res 36:1827-1837.

Deubel H, Schneider WX (2003) Delayed saccades, but not delayed manual aiming movements, require visual attention shifts. Ann N Y Acad Sci 1004:289-296.

Deubel H, Schneider WX, Paprotta I (1998) Selective dorsal and ventral processing: evidence for a common attentional mechanism in reaching and perception. Vis Cogn 5:81-107.

Dickinson AR, Calton JL, Snyder LH (2003) Nonspatial saccade-specific activation in area LIP of monkey parietal cortex. J Neurophysiol 90:2460 2464.

Fischer B, Boch R (1981a) Enhanced activation of neurons in prelunate cortex before visually guided saccades of trained rhesus monkeys. Exp Brain Res 44:129-137.

Fischer B, Boch R (1981b) Selection of visual targets activates prelunate cortical cells in trained rhesus monkey. Exp Brain Res 41:431-433.

Fujii N, Mushiake H, Tanji J (2002) Distribution of eye- and armmovement-related neuronal activity in the SEF and in the SMA and PreSMA of monkeys. J Neurophysiol 87:2158-2166.

Funahashi S, Bruce CJ, Goldman-Rakic PS (1989) Mnemonic coding of visual space in the monkey's dorsolateral prefrontal cortex. J Neurophysiol 61:331-349.

Goldberg ME, Bushnell MC (1981) Behavioral enhancement of visual responses in monkey cerebral cortex. II. Modulation in frontal eye fields specifically related to saccades. J Neurophysiol 46:773-787.

Hoffman JE, Subramaniam B (1995) The role of visual attention in saccadic eye movements. Percept Psychophys 57:787-795.

Lawrence BM, Snyder LH (2006) Comparison of effector-specific signals in frontal and parietal cortices. J Neurophysiol 96:1393-1400.

Lawrence BM, White RL 3rd, Snyder LH (2005) Delay-period activity in visual, visuomovement, and movement neurons in the frontal eye field. J Neurophysiol 94:1498-1508.

Levy I, Schluppeck D, Heeger DJ, Glimcher PW (2007) Specificity of human cortical areas for reaches and saccades. J Neurosci 27:4687-4696.

Mazer JA, Gallant JL (2003) Goal-related activity in V4 during free viewing visual search. Evidence for a ventral stream visual salience map. Neuron 40:1241-1250.
Moore T, Armstrong KM (2003) Selective gating of visual signals by microstimulation of frontal cortex. Nature 421:370-373.

Moore T, Chang MH (2009) Presaccadic discrimination of receptive field stimuli by area V4 neurons. Vision Res 49:1227-1232.

Murthy A, Thompson KG, Schall JD (2001) Dynamic dissociation of visual selection from saccade programming in frontal eye field. J Neurophysiol 86:2634-2637.

Mushiake H, Fujii N, Tanji J (1996) Visually guided saccade versus eye-hand reach: contrasting neuronal activity in the cortical supplementary and frontal eye fields. J Neurophysiol 75:2187-2191.

Quian Quiroga R, Snyder LH, Batista AP, Cui H, Andersen RA (2006) Movement intention is better predicted than attention in the posterior parietal cortex. J Neurosci 26:3615-3620.

Rizzolatti G, Riggio L, Sheliga BM (1994) Space and selective attention. In: Attention and performance XV: conscious and unconscious information processing (Umiltà C, Moscovitch M, eds), pp 231-265. Cambridge, MA: MIT.

Sato TR, Watanabe K, Thompson KG, Schall JD (2003) Effect of targetdistractor similarity on FEF visual selection in the absence of the target. Exp Brain Res 151:356-363.

Schall JD, Hanes DP, Thompson KG, King DJ (1995) Saccade target selection in frontal eye field of macaque. I. Visual and premovement activation. J Neurosci 15:6905-6918.

Shepherd M, Findlay JM, Hockey RJ (1986) The relationship between eye movements and spatial attention. Q J Exp Psychol A 38:475-491.

Snyder LH, Batista AP, Andersen RA (1997) Coding of intention in the posterior parietal cortex. Nature 386:167-170.

Snyder LH, Batista AP, Andersen RA (2000) Intention-related activity in the posterior parietal cortex: a review. Vision Res 40:1433-1441.

Tehovnik EJ, Sommer MA, Chou IH, Slocum WM, Schiller PH (2000) Eye fields in the frontal lobes of primates. Brain Res Brain Res Rev 32:413-448.

Thompson KG, Hanes DP, Bichot NP, Schall JD (1996) Perceptual and motor processing stages identified in the activity of macaque frontal eye field neurons during visual search. J Neurophysiol 76:4040-4055.

Thompson KG, Bichot NP, Schall JD (1997) Dissociation of visual discrimination from saccade programming in macaque frontal eye field. J Neurophysiol 77:1046-1050.

Thompson KG, Biscoe KL, Sato TR (2005) Neuronal basis of covert spatial attention in the frontal eye field. J Neurosci 25:9479-9487.

Tolias AS, Moore T, Smirnakas SM, Tehovnik EJ, Siapas AG, Schiller PH (2001) Eye movements modulate visual receptive fields of V4 neurons. Neuron 29:757-767. 